\title{
Visualization of topological objects in the deconfinement phase of pure $\mathrm{QCD}$ *
}

\author{
Markus Feurstein, Harald Markum and Stefan Thurner \\ Institut für Kernphysik, TU Wien, Wiedner Hauptstraße 8-10, A-1040 Vienna, Austria
}

In the last years it turned out that instantons and monopoles have a certain local correlation in four-dimensional QCD. It was demonstrated by several groups independently and by different methods that at the locations of instantons also monopoles in the maximal abelian projection can be found with enhanced probability. Further we observed such nontrivial correlation functions in the deconfinement phase. We continue our visualization project to analyze several specific gauge-field configurations.

There are two different types of topological objects which seem to be important candidates for the confinement mechanism: color magnetic monopoles and instantons. In lattice calculations we demonstrated that color magnetic monopoles and instantons are correlated on realistic gaugefield configurations [1]. Similar phenomena were discussed by other groups on semiclassical configurations [2]. This might indicate that both confinement mechanisms have a common origin and that both approaches can be united. It is believed that instantons and also monopoles can explain chiral symmetry breaking [3, , 4 . In this contribution we study the origin of the relation between the topological objects by analyzing the correlation functions per gauge-configuration and by visualizing the topological structure by means of $3 \mathrm{D}$ graphics.

To investigate monopole currents we project $S U(N)$ onto its abelian degrees of freedom, such that an abelian $U(1)^{N-1}$ theory remains [5]. We employ the so-called maximum abelian gauge being most favorable for our purposes. For the definition of the monopole currents $m_{i}(x, \mu), i=1, \ldots, N$, we use the standard method [6]. From the monopole currents we define the local monopole density as $\rho(x)=$ $\frac{1}{4 N V_{4}} \sum_{\mu, i}\left|m_{i}(x, \mu)\right|$.

There exist several definitions of the topological charge on the lattice. The field theoretic prescrip-

\footnotetext{
*Supported in part by FWF under Contract No. P11456 Talk presented by H. Markum
}

tions are a straightforward discretization of the continuum expression. To get rid of the renormalization constants we apply the "CabbiboMarinari cooling method" which smooths the quantum fluctuations of a gauge-field. Other topological charge operators can be obtained from the geometric definitions. The discrete set of link variables is interpolated to the continuum and then the topological charge is calculated directly. Concerning the correlation between monopoles and instantons it was shown in [7] that the geometric Lüscher charge definition yields qualitatively the same results as the field theoretic prescriptions. Therefore we employ in these studies of the topological charge density $q(x)$ the field theoretic plaquette and hypercube prescription [8]. To measure correlations between topological quantities we calculate functions of the type

$q(0) q(r), \rho(0)|q(r)|$

per gauge-field configuration.

Our simulations were performed on a $12^{3} \times 4$ lattice with periodic boundary conditions using the Metropolis algorithm. The observables were studied both in the confinement and the deconfinement phase of pure $\mathrm{SU}(2)$ theory at inverse gluon coupling $\beta=4 / g^{2}=2.25$ and 2.4 , respectively. For each run we made 100 measurements, separated by 100 iterations. In previous work we found a spatial relation between instantons and monopoles by averaging the correlation between the topological charge density and the monopole density over an ensemble of gauge-fields 
Confinement
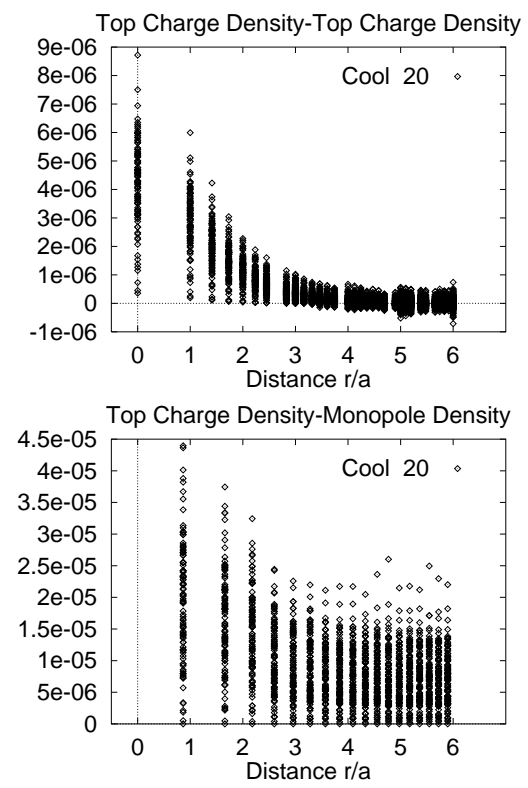

Deconfinement
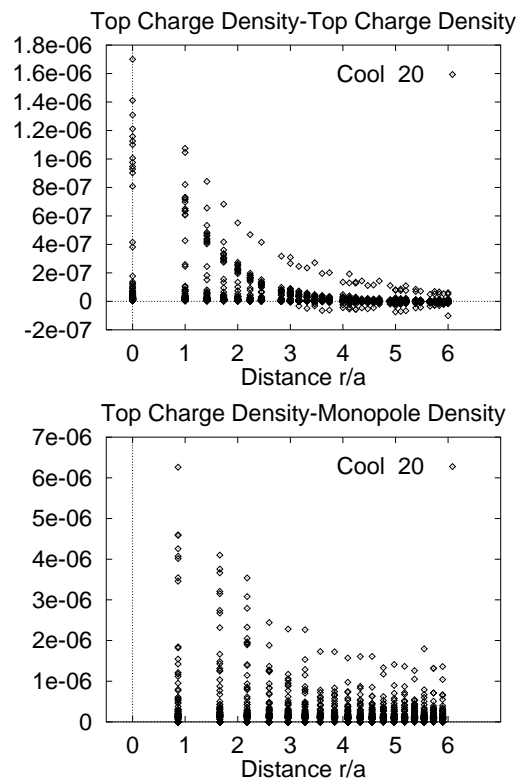

Figure 1. Auto-correlation functions of the topological charge density after 20 cooling steps for 100 independent configurations in both phases (top). In contrast to the confinement phase only $15 \%$ of the configurations carry a topological charge in the deconfinement phase. The corresponding $\rho|q|$-correlations are displayed in both phases (bottom). All configurations with nonvanishing $q q$-auto-correlation give rise to a nontrivial $\rho|q|$-correlation.

[7]. The coexistence between both topological objects turned out to be hardly affected by cooling and behaved similarly in both phases of the theory. In this contribution we study the origin of the nontrivial correlation between monopoles and instantons by considering single gauge-fields. In particular we are interested in the reason for the similarity in both phases.

Fig. 1 presents the auto-correlations of the topological charge in the plaquette definition and the $\rho|q|$-correlations after 20 cooling steps for 100 independent configurations. In the confinement phase the auto-correlation functions have many different amplitudes reflecting a large variety of topologically nontrivial configurations. Also the corresponding monopole-instanton correlations show many different amplitudes. In the deconfinemet phase only about $15 \%$ of the auto-correlation functions are nontrivial. All of these configurations give rise to a nontrivial $\rho|q|$ correlation. This indicates that the relation between monopoles and instantons found on gauge average also holds for single configurations.

In Fig. 2 we visualize this relationship by directly displaying clusters of topological charge and by drawing monopole loops for fixed $t$ or $x$ of a specific configuration in the deconfinement phase. For any value of the topological charge density $q(x)>0.005$ a light dot and for $q(x)<-0.005$ a dark dot is plotted. Monopole loops are represented by lines. This configuration has a total topological charge of $Q=2$. One observes a timelike monopole loop passing through each instanton.

To summarize, we analyzed the topological structure of the $S U(2)$ vacuum at finite temperature in both phases of the theory. The autocorrelation functions of the topological charge 
density are nontrivial after 20 cooling steps reflecting the existence of instantons. Correlation functions between the topological charge density and the monopole density are hardly affected by cooling indicating a close spatial relation between instantons and monopoles. This observation also holds in the deconfinement phase.

Studying the origin of the coexistence between monopoles and instantons in more detail we computed correlation functions per configuration. In the deconfinement phase only approximately 15 $\%$ of the configurations carry a topological charge and all of these configurations give rise to nonvanishing $\rho|q|$-correlations. Visualization demonstrated that across the transition and after cooling instantons are squeezed in time direction and are accompanied by timelike monopoles. It will be interesting to check this result by other methods not relying on cooling like inverse blocking.

\section{REFERENCES}

1. S. Thurner, H. Markum and W. Sakuler, Proceedings of Confinement 95, Osaka 1995, eds. H. Toki et al. (World Scientific, 1996) 77 (hep-th/9506123); S. Thurner, M. Feurstein, H. Markum and W. Sakuler, Phys. Rev. D54 (1996) 3457; M. Feurstein, H. Markum and S. Thurner, Nucl. Phys. B (Proc. Suppl.) 53 (1997) 553.

2. M.N. Chernodub and F.V. Gubarev, JETP Lett. 62 (1995) 100; A. Hart and M. Teper, Phys. Lett. B371 (1996) 261; V. Bornyakov and G. Schierholz, Phys. Lett. B384 (1996) 190; R.C. Brower, K.N. Orginos and ChungI Tan, Phys. Rev. D55 (1997) 6313.

3. E.V. Shuryak, Nucl. Phys. B302 (1988) 559.

4. O. Miyamura, Nucl. Phys. B (Proc. Suppl.) $42(1995) 538$.

5. G. 't Hooft, Nucl. Phys. B190 (1981) 455.

6. A.S. Kronfeld, G. Schierholz and U.-J. Wiese, Nucl. Phys. B293 (1987) 461.

7. M. Feurstein, H. Markum and S. Thurner, Phys. Lett. B396 (1997) 203.

8. P. Di Vecchia, K. Fabricius, G.C. Rossi and G. Veneziano, Nucl. Phys. B192 (1981) 392; Phys. Lett. B108 (1982) 323; Phys. Lett. B249 (1990) 490.
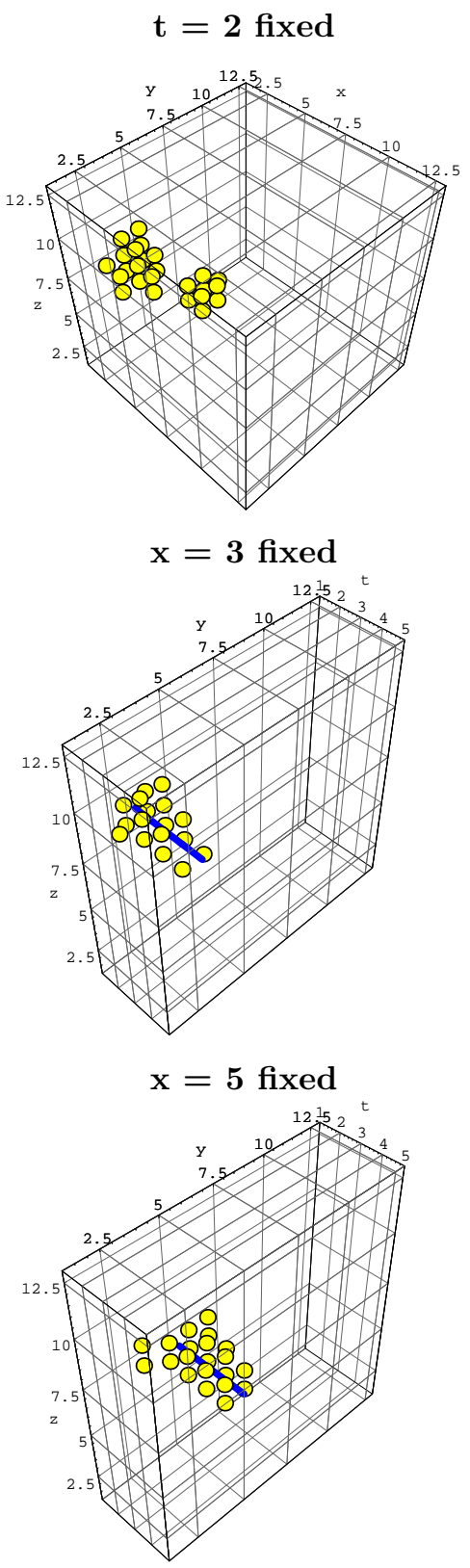

Figure 2. Three-dimensional visualization of a four-dimensional configuration in the deconfinement at 20 cooling steps with topological charge $Q=2$. The dots indicate the topological charge density $|q(x)|>0.005$ and the lines represent monopole loops. 